\title{
Mid-infrared imaging of brown dwarfs in binary systems ${ }^{\star}$ (Research Note)
}

\author{
K. Geißler ${ }^{1,2}$, G. Chauvin ${ }^{1,3}$, and M. F. Sterzik ${ }^{1}$ \\ 1 European Southern Observatory, Alonso de Cordova 3107, Vitacura, Santiago, Chile \\ e-mail: geissler@mpia-hd.mpg.de; gael.chauvin@obs.ujf-grenoble.fr; msterzik@eso.org \\ 2 Max-Planck-Institut für Astronomie, Königstuhl 17, 69117 Heidelberg, Germany \\ 3 Laboratoire d'Astrophysique, Observatoire de Grenoble, UJF, CNRS, BP 53, 38041 Grenoble Cedex 9, France \\ Received 5 July 2007 / Accepted 8 December 2007
}

\section{ABSTRACT}

\begin{abstract}
Context. Brown dwarfs exhibit complex atmospheric signatures, and their properties are highly sensitive to effective temperature, surface gravity, and metallicity. Several physical properties of brown dwarfs in binary systems can be well inferred from the primary, and therefore allow one to better constrain their atmospheres.

Aims. We want to constrain atmospheric models of brown dwarfs in binary systems using narrow-band mid-infrared photometry.

Methods. High spatial resolution and sensitivity is required to resolve the components. Therefore we have obtained deep mid-infrared images of four close binary systems with brown dwarf companions using VISIR at the Very Large Telescope (VLT) in three narrowband filters at $8.6,10.5$ and $11.25 \mu \mathrm{m}$.

Results. Three brown dwarf companions (GJ 229 B, HD 130948 BC and HR 7329 B) were detected at $8.6 \mu \mathrm{m}$. HD 130948 BC was also observed at $10.5 \mu \mathrm{m}$. Finally, we establish upper flux limits for the other narrow band filters with null detections.

Conclusions. Our results, are in general, compatible with previous observations and model expectations for these objects. For HD $130948 \mathrm{BC}$, we conclude that there is photometric variability on a significance level of $2.8 \sigma$ based on repeated observations. The bandpass around $10.5 \mu \mathrm{m}$ appears specifically well suited for variability studies, and we speculate that either inhomogeneities in the atmospheric $\mathrm{NH}_{3}$ distribution, or silicate absorption, might cause its time-variability.
\end{abstract}

Key words. stars: low-mass, brown dwarfs - stars: fundamental parameters

\section{Introduction}

Brown dwarfs (BDs) bridge the gap in mass between low-mass stars and giant planets. Hundreds of them have been discovered in the past decade, mainly in wide-field optical (SDSS, Stoughton et al. 2002) and near-infrared (e.g., 2MASS, DENIS; Cutri et al. 2003; Epchtein et al. 1997) surveys. Two main classes of BDs emerged based on their optical and infrared spectral properties, the L-dwarfs and T-dwarfs. They typically cover effective temperature ranges of $T_{\text {eff }} \approx 2200-1400 \mathrm{~K}$ and $T_{\text {eff }} \approx 1400-700 \mathrm{~K}$, respectively. The modeling of atmospheres cooler than $T_{\text {eff }} \leq 2000 \mathrm{~K}$ is a challenge, because it must include an appropriate treatment of a plethora of molecular opacity's and dust processes (formation, condensation, size distribution and mixing). The most recent atmosphere models include additional properties such as age (gravity) and metallicity, and seem to reproduce the spectral signatures and the infrared colours of L and T dwarfs reasonably well. Only the L-T transition, occurring around a relatively narrow temperature range $T_{\text {eff }} \approx 1300-1400 \mathrm{~K}$, remains problematic (for a discussion of state-of-the-art models see Burrows et al. 2006).

As an additional complication, the determination of distances, ages and metallicity for field BDs remains difficult and may be the cause of various uncertainties in their physical parameters. In that sense, BD companions of nearby stars are extremely interesting, as the system characteristics are well known thanks to the bright primary star. For these systems the influence

\footnotetext{
* Based on observations made with ESO Telescopes at the Paranal Observatories under programme IDs 076.C-0556(A) and 077.C0438(A).
}

of age and metallicity on the spectral signatures of substellar objects can be explored with high accuracy.

Initially, most BD studies were conducted in the optical and near-infrared. However, with the recent development of midinfrared observing techniques and instruments from the ground or from space, this spectral region became essential because numerous molecular lines, including dominant absorption bands of $\mathrm{CH}_{4}, \mathrm{CO}, \mathrm{H}_{2} \mathrm{O}$ and $\mathrm{NH}_{3}$, are present. The Spitzer Space Telescope has provided a wealth of high-quality photometry and low- and medium resolution spectroscopy of $\mathrm{L}$ and $\mathrm{T}$ dwarfs in the 5-20 $\mu \mathrm{m}$ regime (see, e.g. Leggett et al. 2007; Cushing et al. 2006; Mainzer et al. 2007). They enable one to probe vertical mixing, clouds and non-equilibrium chemistry around the $\mathrm{L}-\mathrm{T}$ boundary in unprecedented detail.

The power of sensitive, ground-based, high spatial resolution, mid-infrared imaging has recently been demonstrated for the close BD binary companion $\epsilon$ Indi Ba and Bb (see Sterzik et al. 2005). The relative photometry between both components allows one to derive effective temperatures independently from the determinations in near-infrared. As the distance is well known for the primary, the absolute photometry constrains radii and bolometric corrections, in contrast to Spitzer observations (Roellig et al. 2004) that suffered from insufficient angular resolution to resolve both components (Spitzer diffraction limit at $10 \mu \mathrm{m}$ is $\sim 3^{\prime \prime}$ ).

In order to continue our effort to constrain atmospheric and evolutionary models of BDs, we have therefore conceived a mini-survey of close BD companions using the mid-infrared imager VISIR at the VLT. Section 2 reviews the target's properties and describes our observations. Section 3 describes our data 
Table 1. Target properties.

\begin{tabular}{|c|c|c|c|c|c|c|c|c|}
\hline Object & $\begin{array}{r}\pi \\
{[\mathrm{mas}]}\end{array}$ & $\begin{array}{r}\text { Age } \\
{[\mathrm{Gyr}]}\end{array}$ & $\overline{[\overline{[M / H]}}$ & $\begin{array}{r}\text { Separ. } \\
\text { [arcsec] }\end{array}$ & $\begin{array}{r}\mathrm{PA} \\
\text { [degree] }\end{array}$ & $\begin{array}{r}\text { Spectral } \\
\text { type B }\end{array}$ & $\begin{array}{l}T_{\text {eff }} \\
{[\mathrm{K}]}\end{array}$ & $\log g$ \\
\hline$\overline{\text { GJ } 229(1)}$ & 173.19 & $0.2(1)$ & $-0.1-0.5(4)$ & $7.761 \pm 0.007$ (3) & $163.5 \pm 0.1$ & T6.5 & $950 \pm 80(4)$ & $\begin{array}{l} \pm 0.5(4) \\
<35(2)\end{array}$ \\
\hline D & 55.73 & $\begin{array}{r}0.030(2) \\
3-0.8(6-8)\end{array}$ & $\begin{array}{l}-0.5(2) \\
0.0(10)\end{array}$ & 10 & & & $\begin{array}{l}100(2) \\
75(9)\end{array}$ & $5(2)$ \\
\hline R 7 & 20.98 & $12(12)$ & & $4.17 \pm 0.05$ & $166.8 \pm 0.2$ & M7/M8 (11) & $00(11)$ & \\
\hline HR 7672 (13) & 56.60 & 1.-3.(13) & $0.02(10)$ & $0.794 \pm 0.005$ & $157.3 \pm 0.6$ & $\mathrm{~L} 4.5 \pm 1.5(13)$ & $1680 \pm 170$ & \\
\hline
\end{tabular}

^ Binary brown dwarf, see text. Reference: 1) Nakajima et al. (1995); 2) Leggett et al. (2002); 3) Golimowski et al. (1998); 4) Saumon et al. (2000); 5) Potter et al. (2002); 6) Gaidos (1998); 7) Gaidos et al. (2000); 8) Fuhrmann (2004); 9) Goto et al. (2002); 10) Valenti \& Fischer (2005); 11) Lowrance et al. (2000); 12) Zuckerman et al. (2001); 13) Liu et al. (2002).

reduction and analysis process. Section 4 presents the results of our survey, i.e. the photometry and the astrometry of the detected companions and the sensitivity limits obtained. Finally, Sect. 5 compares our results with the predictions of cool atmosphere models, to discuss their applicability and limitations.

\section{Target properties and observation}

For our target selection, we have only considered the confirmed members of binary (or multiple) systems with known distances, as their primaries are well characterized in terms of metallicity and age. Only BD companions with expected mid-IR fluxes stronger than $1 \mathrm{mJy}$ and separations larger then $0.5^{\prime \prime}$ were selected, in order to fully adapt and exploit the sensitivity and spatial resolution of the mid-infrared imager VISIR at the VLT. We finished with a short list of four systems: GJ 229, HD 130948, HR 7329 and HR 7672. Their main characteristics are summarized in Table 1.

- GJ 229 B is the first unambiguous BD discovered (Nakajima et al. 1995). Later on, orbital motion was detected by Golimowski et al. (1998), who observed GJ 229 B at three epochs spread over approximately one year using HST's Wide Field Planetary Camera 2 (WFPC2). Matthews et al. (1996) derived an effective temperature of $\sim 900 \mathrm{~K}$ from the measured broadband spectrum of GJ $229 \mathrm{~B}$, assuming a radius equal to that of Jupiter. The same effective temperature was obtained by Leggett et al. (1999) by comparing colours and luminosity to evolutionary models developed by Burrows et al. (1997). In general model, spectra for GJ 229 B (Marley et al. 1996; Allard et al. 1996) reproduce the overall energy distribution fairly well and agree with $T_{\text {eff }}=950 \mathrm{~K}$.

- HD 130948 BC is a binary brown dwarf companion detected by Potter et al. (2002). The separation between the two companions is $(0.134 \pm 0.005)^{\prime \prime}$ at $\mathrm{PA}=(317 \pm 1)^{\circ}$. Both companions have the same spectral type (L4 \pm 1$)$ and effective temperatures $\left(T_{\mathrm{eff}}=1900 \pm 75 \mathrm{~K}\right)$ (Goto et al. 2002).

- HR 7329 B is a BD companion detected by Lowrance et al. (2000) at a separation of $4^{\prime \prime}$ from the early-type star HR 7329A, a member of the $\beta$ Pictoris moving group (Zuckerman et al. 2001). Its optical spectrum points towards a spectral type of M7/M8 and an effective temperature of 2405 to $2770 \mathrm{~K}$ for this young substellar companion. Guenther et al. (2001) show evidence that the source is a comoving companion.

- HR 7672 B, a common proper motion companion to the variable star HR 7672 A, was reported by Liu et al. (2002). They inferred an effective temperature of $T_{\text {eff }}=1510-1850 \mathrm{~K}$ for HR 7672 B and estimated an age of 1-3 Gyr for the system.
All targets were observed using VISIR (Lagage et al. 2004) mounted at the UT3 (Melipal) with the filters PAH1 $\left(\lambda_{\text {cen }}=8.59 \mu \mathrm{m}, \Delta \lambda=0.42 \mu \mathrm{m}\right)$, PAH2 $\left(\lambda_{\text {cen }}=11.25 \mu \mathrm{m}\right.$, $\Delta \lambda=0.59 \mu \mathrm{m})$ and $\operatorname{SIV}\left(\lambda_{\text {cen }}=10.49 \mu \mathrm{m}, \Delta \lambda=0.16 \mu \mathrm{m}\right)$. A nominal pixelscale of $0.075^{\prime \prime}$ was used during all observations, and standard chopping and nodding techniques were employed, with a chop-throw amplitude of $6^{\prime \prime}$ and $8^{\prime \prime}$ for GJ 229, and a chopping frequency of $0.25 \mathrm{~Hz}$. The nodding direction was chosen parallel to the chopping direction and, consequently, with an equal nodding to chopping amplitude.

To ensure that the primary and the companion are within the FoV during chopping and nodding, the system was aligned horizontally on the detector. At the same time, this simplifies the reduction process, since the shifting and adding of the frames can be done using the brighter primary star. A summary of the observing $\log$ is given in Table 2, including the mean airmass during the observing run and the total integration time.

\section{Data analysis}

\subsection{Data reduction}

The reduction was done using self-written IDL routines for badpixel replacement and for the shifting and adding of the frames. Bad-pixels were replaced by the mean value of the surrounding pixels within a box of $9 \times 9$ pixel, before subtracting $(A-B)$ nodding positions. In the following, the relative shifts between the frames of one data-set were calculated via cross-correlation of the bright primary, before the frames were averaged. Since the VISIR detector was affected by randomly triggered stripes during part of the observations, a destriping technique developed by Pantin et al. (2007) was applied to the final co-added images.

\subsection{Aperture photometry}

Standard aperture photometry was used to determine the relative photometry of all detected BDs. Using IDL ATV routines, a curve-of-growth method was applied to the brown dwarf companions to obtain the apertures where the signal-to-noise ratio is maximised. In the following, those apertures were used for the primary as well as for the standard stars. Thus the count-rate to flux conversion factor was determined and relative photometry obtained. The variation of the count-rate to flux conversion factors with aperture radius was screened for at least 3 consecutive aperture radii between 4 and 7 pixels (corresponding to radii of $0.3^{\prime \prime}$ to $0.525^{\prime \prime}$ ). At $10 \mu \mathrm{m}$ the VISIR diffraction limit is $\sim 0.3^{\prime \prime}$, the chosen aperture radii are of the order of twice the diffraction limit. To calibrate the flux values different standard stars ${ }^{1}$, observed before and after the targets, were used. The error bar

1 Taken from the list of Cohen et al. (1999). 
Table 2. Observing Log.

\begin{tabular}{|c|c|c|c|c|c|c|c|c|c|c|}
\hline Object & Filter & $\begin{array}{r}\text { UT date } \\
\mathrm{dd} / \mathrm{mm} / \mathrm{yr}\end{array}$ & Airmass & $\begin{array}{r}\text { DIMM }^{a} \\
\text { seeing ["] }\end{array}$ & $\begin{array}{r}\text { Humidity } \\
{[\%]}\end{array}$ & $\begin{array}{r}\text { DIT } \\
{[\mathrm{s}]}\end{array}$ & NDIT & $\begin{array}{r}\text { \# of } \\
\operatorname{nods}^{c}\end{array}$ & $\begin{array}{r}\text { Int. Time }^{d} \\
{[\mathrm{~s}]}\end{array}$ & Calibrator \\
\hline \multirow[t]{5}{*}{$\overline{\text { GJ } 229}$} & PAH1 & $08 / 01 / 06^{b}$ & 1.109 & 0.91 & $17-50$ & 0.016 & 123 & 12 & 2172.7 & HD 26967, HD 75691 \\
\hline & & $10 / 02 / 06^{b}$ & 1.195 & 0.79 & $23-55$ & 0.016 & 123 & 18 & 3259.0 & HD 41047, HD 75691 \\
\hline & $\mathrm{PAH} 2$ & $03 / 02 / 06^{b}$ & 1.044 & 0.83 & $20-40$ & 0.008 & 246 & 6 & 1086.3 & HD 41047, HD 26967 \\
\hline & & $10 / 02 / 06$ & 1.110 & 0.84 & $23-55$ & 0.008 & 246 & 6 & 1086.3 & HD 26967, HD 75691 \\
\hline & SIV & $10 / 02 / 06$ & 1.022 & 0.95 & $23-55$ & 0.04 & 48 & 11 & 1943.0 & HD 26967, HD 75691 \\
\hline \multirow[t]{5}{*}{ HD 130948} & PAH1 & 09-10/07/06 & 1.524 & 0.67 & $6-8$ & 0.020 & 98 & 22 & 3967.0 & HD 133774, HD 99167 \\
\hline & PAH2 & $11 / 07 / 06^{b}$ & 1.541 & 0.91 & $7-10$ & 0.010 & 197 & 22 & 3987.3 & HD 149009 \\
\hline & & $04-05 / 08 / 06^{b}$ & 1.734 & 0.71 & 7 & 0.010 & 197 & 11 & 1993.6 & HD 149009, HD 145897 \\
\hline & SIV & 03/08/06 & 1.627 & 0.86 & $5-8$ & 0.040 & 48 & 11 & 1943.0 & HD 145897 \\
\hline & & $05 / 08 / 06$ & 1.606 & 0.62 & $6-11$ & 0.040 & 48 & 11 & 1943.0 & HD 145897 \\
\hline \multirow[t]{3}{*}{$\overline{\text { HR } 7329}$} & PAH1 & $07 / 06 / 06$ & 1.182 & 1.05 & $4-12$ & 0.020 & 98 & 22 & 3967.0 & HD 178345 \\
\hline & $\mathrm{PAH} 2$ & $21 / 05 / 06$ & 1.153 & 1.36 & $7-19$ & 0.008 & 246 & 11 & 1991.6 & HD 178345 \\
\hline & SIV & 07/06/06 & 1.175 & 0.88 & $4-12$ & 0.040 & 48 & 22 & 3886.1 & HD 178345 \\
\hline \multirow[t]{5}{*}{ HR 7672 } & PAH1 & $10 / 07 / 06^{b}$ & 1.341 & 0.76 & $6-8$ & 0.020 & 98 & 11 & 1983.5 & HD 189695, HD 149009 \\
\hline & & $12 / 07 / 06^{b}$ & 1.405 & 0.88 & $6-11$ & 0.016 & 123 & 11 & 1991.6 & HD 189695, HD 178345 \\
\hline & PAH2 & $10 / 07 / 06^{b}$ & 1.385 & 0.81 & $6-8$ & 0.010 & 197 & 11 & 1993.6 & HD 189695, HD 220954 \\
\hline & & $13 / 07 / 06^{b}$ & 1.693 & 0.78 & $5-8$ & 0.010 & 197 & 11 & 1993.6 & HD 198048, HD 217902 \\
\hline & SIV & $13 / 07 / 06^{b}$ & 1.356 & 0.82 & $5-8$ & 0.040 & 48 & 15 & 2649.6 & HD 178345, HD 198048 \\
\hline
\end{tabular}

a) In $V$ band at $550 \mathrm{~nm}, b$ ) data showed stripes, $c) 23$ chops per nod, $d$ ) $t=\operatorname{DIT} \times \operatorname{NDIT} \times \#$ of chops $\times \#$ of nods $\times 4$.

estimation of the flux calibration is derived from the flux variations of the source measured in different apertures and from different standard stars. In cases where two independent measurements were taken, an average of the measured fluxes is quoted in Table 4.

As already mentioned, a destriping technique was applied to the final images to clean it of random stripes and thereby improve the image quality. To estimate the impact of the destriping on the photometry of the BDs, we performed the aperture photometry before and after the destriping process. In those cases in which the source is not located close to a stripe, no influence is noticeable. In contrast in the cases where the BD is close to a stripe, a decrease in the measured count-rate, and consequently in flux, of the BD in the destriped images is perceivable. Nevertheless, this effect is expected, since the stripes in the "raw" images fall within the aperture radii and lead to an overestimation of the count-rate and therefore of the flux.

\subsection{Detection limits}

To estimate the detection limits as a function of angular separation two approaches were explored. The standard deviation of the intensities within a 1 pixel wide annulus at a given radius was determined, as well as the standard deviation within a box of $5 \times 5$ pixels along a random radial direction. Using the obtained noise estimate, the contrast with respect to the peak intensity of the primary was calculated (see, e.g., Fig. 3). The detection limits delivered by both methods are in good agreement. Additionally, to further test the derived detection limits, artificial companions, with fluxes varying between 2 and $10 \mathrm{mJy}$, were placed within the data at separations between 1 and 5". The limiting fluxes of the re-detectable artificial companions match the previously derived detection limits. Up to a separation of $\sim 1.5^{\prime \prime}$ the detection limit is dominated by the photon noise of the central star, and at larger separation the background noise from the atmosphere and the instrument limits our detections.
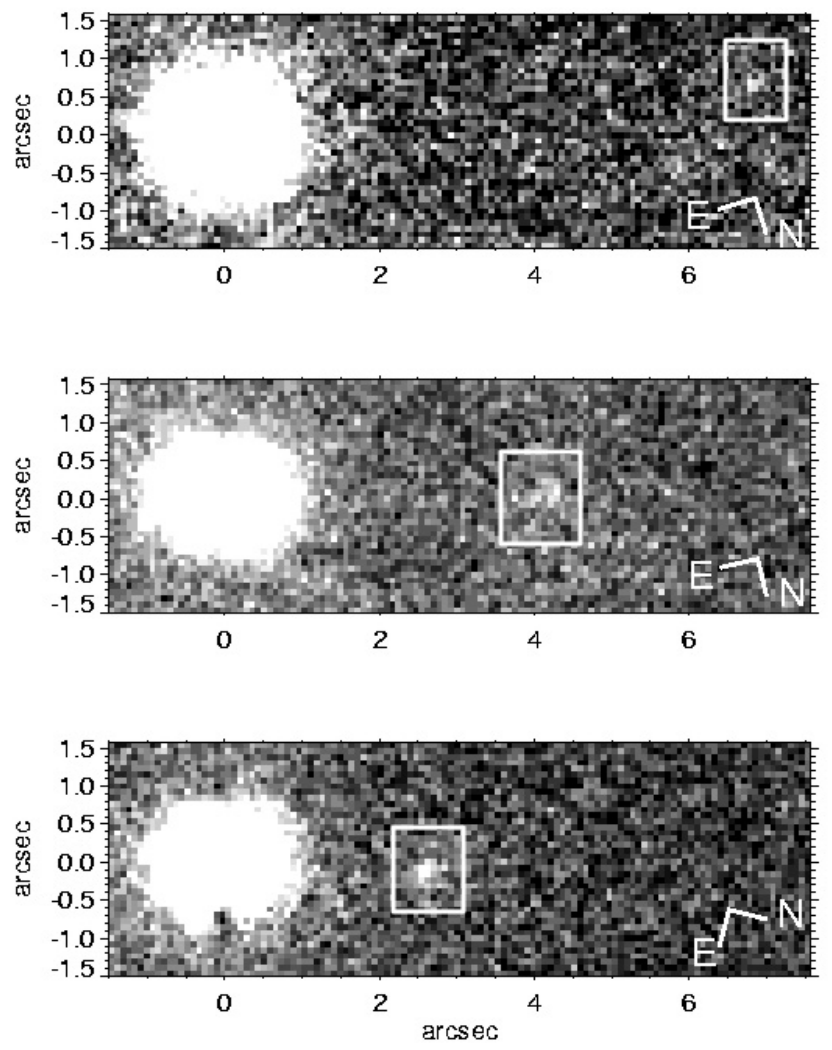

Fig. 1. VISIR detection images of GJ 299B at 6.8" (upper), HR 7329 at $\sim 4.3^{\prime \prime}$ (middle) and HD 130948 BC at $\sim 2.5^{\prime \prime}$ (bottom) at $8.6 \mu \mathrm{m}$. A $\sigma$ filter with a box width of 5 pixel has been applied to all images. Furthermore, the N-E orientation of the data is over indicated in the lower right corner of the image.

\section{Results}

Three of the four brown dwarfs were detected in PAH1, namely GJ 229 B, HR 7329 B and HD 130948 BC (see Fig. 1), while only HD 130948 BC could be detected in SIV. Note that 

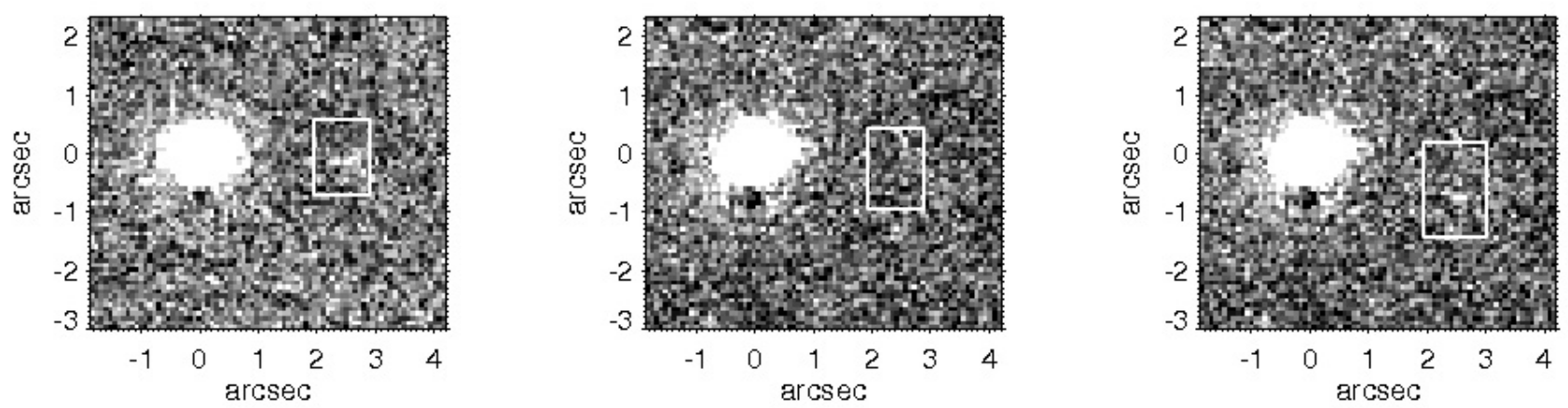

Fig. 2. VISIR images of HD 130948 in the SIV filter taken on the 5th of August (left) and on the 3rd of August (middle). The binary brown dwarf companion was detected in the data from the 5th of August and is marked by a box in the leftmost image. The flux was measured to be $5.7 \pm 0.4 \mathrm{mJy}$. In the data set from the 3rd of August the companion was not detected. Its approximate location is at the same position as in the left image, and also marked by a box. The right image shows the data from the 3rd of August, in which an artificial companion of $4 \mathrm{mJy}$ had been placed. The artificial companion is located somewhat below the expected position of the real companion.

Table 3. Separations and position angles of the detected brown dwarfs.

\begin{tabular}{lrrr}
\hline \hline Object & UT date & Sep. [arcsec] & PA [ $\left.{ }^{\circ}\right]$ \\
\hline GJ 229 & $10 / 02 / 06$ & $6.78 \pm 0.05$ & $168.4 \pm 0.9$ \\
HD 130948 & $09 / 07 / 06$ & $2.54 \pm 0.05$ & $103.9 \pm 2.4$ \\
HR 7329 & $07 / 06 / 06$ & $4.17 \pm 0.11$ & $167.2 \pm 1.4$ \\
\hline
\end{tabular}

HD 130948 BC, a binary brown dwarf, was not resolved in our observations. HR 7672 B could not be detected in any of the filters. While the resolution of VISIR is sufficient to separate the brown dwarf and the primary ( $\sim 0.79^{\prime \prime}$ Liu et al. 2002; assuming negligible orbital motion), the data quality in PAH1 and SIV is low. The PSF of the primary is elongated, affecting the area in which the brown dwarf is expected, and thus adding noise.

In Table 3 the measured separations and position angles of the detected brown dwarfs are given. To obtain the separation as well as the position angle of the brown dwarfs relative to their primaries, the pixelscale and $\mathrm{N}$-orientation provided in the image header were used. Golimowski et al. (1998), used the HST's Wide Field Planetary Camera 2 (WFPC2) to observe GJ 229 B at three epochs, which were spread over approximately one year. Orbital motion of GJ 229 B was detected and a relative change of separation of $(0.088 \pm 0.010)^{\prime \prime}$ per year was measured. In the last 10 years, from November 1996 to February 2006, the separation between GJ $229 \mathrm{~A}$ and B changed by $(0.894 \pm 0.05)^{\prime \prime}$, resulting in an average change of separation of $(0.097 \pm 0.005)^{\prime \prime}$ per year. For HD 130948, only a minor change of separation is observable. From February 2001 to July 2006 the separation between HD 130948 A and BC decreased by $(0.09 \pm 0.05)^{\prime \prime}$. In the case of HR 7329 no orbital motion was observable. In Table 4 the obtained fluxes for the primary stars and the brown dwarfs, and the upper limits for the non-detections, are listed. In the case of HD 130948 BC the flux measured in the data set from the 5th of August is quoted, as well as the upper limit obtained on the 3rd of August. While the observations of HD 130948 BC in SIV have been carried out at two different epochs, on the 3rd and the 5th of August, the object was only detectable in the second data set (see Fig. 2) with a measured flux of $(5.7 \pm 0.4) \mathrm{mJy}$. The non-detection of HD 130948 BC in the data set from the 3rd of August can not be explained by a discrepancy in the sensitivity limits, see Fig. 3. Both data sets clearly reach the same sensitivity limit. Furthermore, simulations of artificial sources showed that a companion with a flux of $(4 \pm 0.4) \mathrm{mJy}$ (corresponding to a $5 \sigma$ confidence level) would have been detected
Table 4. VISIR photometry of the primaries and brown dwarfs. In cases of non-detections, upper limits are provided. The fluxes are quoted in mJy.

\begin{tabular}{|c|c|c|c|}
\hline Object & 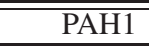 & $\overline{\overline{\text { SIV }}}$ & $\overline{\mathrm{PAH} 2}$ \\
\hline$\overline{\mathrm{GJ}} 229 \mathrm{~A}$ & 1297. (47) & 923. (33.) & 793. (26.) \\
\hline GJ 229 B & $3.2(0.5)$ & 2. $(0.3)^{a}$ & 4. ${ }^{a}(0.9)$ \\
\hline HD 130948 A & 861. (5.) & 605. $(27 .)^{b}$ & 478. (6.) \\
\hline HD 130948 BC & $3.8(0.4)$ & $5.7(0.4)^{b}$ & $1.8(0.2)^{a}$ \\
\hline HD 130948 A & & 553. (27. $)^{c}$ & \\
\hline HD 130948 BC & & 2. $(0.4)^{a, c}$ & \\
\hline HR 7329 A & 524. (19.) & 404. (3.) & 386. (24.) \\
\hline HR $7329 \mathrm{~B}$ & $3.2(2.3)$ & $1.3(0.2)^{a}$ & $2.3(0.2)^{a}$ \\
\hline HR 7672 A & 880. (36.) & 554. (33.) & 519. (14.) \\
\hline
\end{tabular}

a) Limiting background $(1 \sigma), b)$ only 05.08.2006, c) 03.08.2006.

in both data sets. Hence, within $\approx 48$ h HD 130948 BC varied by at least $(1.7 \pm 0.6) \mathrm{mJy}$.

\section{Discussion}

\subsection{Comparison with models}

As a final step we compare our obtained photometry to the models developed by Allard et al. (2001) and Burrows et al. (2006). Using their theoretical spectra, provided online ${ }^{2}$, absolute model fluxes were calculated by integrating the theoretical spectrum over the VISIR filter bandpasses. The object radii $R$, which determine the absolute spectral flux calibration, were obtained from evolutionary calculations by Burrows et al. (1997). In Table 5 the calculated model fluxes are listed. Furthermore, the age and effective temperature combinations for which the object radius was determined are given. From Allard et al. (2001) we employed the AMES-cond and AMES-dusty models, representing the two extreme cases, in which either all dust has disappeared from the atmosphere (AMES-cond) or dust settling throughout the atmosphere is negligible (AMES-dusty). Following Allard et al. (2001) the AMES-dusty models should successfully describe dwarfs with effective temperatures greater than $1800 \mathrm{~K}$, while the AMES-cond models are better suited to describe the atmospheres of dwarfs with $T_{\text {eff }} \leq 1300 \mathrm{~K}$.

\footnotetext{
2 http://perso.ens-lyon.fr/france.allard/ and http:// zenith.as.arizona.edu/burrows/
} 
Table 5. Predicted mid-IR fluxes from different theoretical models. Values consistent within $3 \sigma$ or with the given upper limit were marked in boldface.

\begin{tabular}{|c|c|c|c|c|c|c|c|c|c|}
\hline Object & Reference & $\begin{array}{l}T_{\text {eff }} \\
{[\mathrm{K}]}\end{array}$ & $\begin{array}{r}\log g \\
{\left[\mathrm{~cm} / \mathrm{s}^{2}\right]}\end{array}$ & {$[M / H]$} & $\begin{array}{r}\text { age } \\
\text { Myr }\end{array}$ & $R / R_{\odot}$ & $\begin{array}{l}\text { PAH1 } \\
{[\mathrm{mJy}]}\end{array}$ & $\begin{array}{r}\text { SIV } \\
{[\mathrm{mJy}]}\end{array}$ & $\begin{array}{l}\mathrm{PAH} 2 \\
{[\mathrm{mJy}]}\end{array}$ \\
\hline \multirow[t]{10}{*}{$\overline{\text { GJ } 229 \mathrm{~B}}$} & Allard $^{a}$ & 900 & 5.0 & 0.0 & 200 & 0.122 & 3.30 & 2.97 & 5.06 \\
\hline & Allard $^{a}$ & 1000 & 5.0 & 0.0 & 200 & 0.122 & 4.67 & 4.55 & 6.68 \\
\hline & Allard $^{a}$ & 1000 & 3.0 & 0.0 & 200 & 0.122 & 4.80 & 6.69 & 7.94 \\
\hline & Allard $^{a}$ & 1000 & 3.0 & 0.0 & 30 & 0.133 & 5.70 & 7.95 & 9.44 \\
\hline & Burrows $^{c}$ & 900 & 5.0 & 0.0 & 200 & 0.122 & 3.35 & 3.0 & 4.25 \\
\hline & Burrows $^{c}$ & 900 & 5.0 & -0.5 & 200 & 0.122 & 3.33 & 2.46 & 3.78 \\
\hline & Burrows $^{c}$ & 1000 & 5.0 & -0.5 & 200 & 0.122 & 4.25 & 3.44 & 4.77 \\
\hline & Burrows $^{c}$ & 1000 & 5.0 & -0.5 & 30 & 0.133 & 5.04 & 4.09 & 5.66 \\
\hline & Burrows $^{c}$ & 1000 & 4.5 & -0.5 & 30 & 0.133 & 5.14 & 4.99 & 6.05 \\
\hline & measured & & & & & & $3.2 \pm 0.5$ & $3.2^{1}$ & $6.7^{1}$ \\
\hline \multirow[t]{7}{*}{ HD 130948 BC } & Allard $^{b}$ & 1900 & 5.0 & 0.0 & 800 & 0.091 & 1.65 & 1.25 & 1.13 \\
\hline & Allard $^{b}$ & 1900 & 5.0 & 0.0 & 300 & 0.102 & 2.07 & 1.57 & 1.42 \\
\hline & Allard $^{b}$ & 1900 & 3.5 & 0.0 & 300 & 0.102 & 1.67 & 1.21 & 1.11 \\
\hline & Burrows $^{d}$ & 1900 & 5.0 & 0.0 & 300 & 0.102 & 1.53 & 1.26 & 1.17 \\
\hline & Burrows $^{d}$ & 1900 & 5.0 & -0.5 & 300 & 0.102 & 1.49 & 1.25 & 1.16 \\
\hline & measured $^{2}$ & & & & & & $1.9 \pm 0.3$ & $2.9 \pm 0.3^{3}$ & $1.2^{1}$ \\
\hline & measured $^{2}$ & & & & & & & $1.5^{1}$ & \\
\hline \multirow[t]{6}{*}{ HR $7329 \mathrm{~B}$} & Allard $^{b}$ & 2400 & 4.0 & 0.0 & 12 & 0.193 & 1.06 & 0.91 & 0.79 \\
\hline & Allard $^{b}$ & 2600 & 3.5 & 0.0 & 8 & 0.278 & 2.80 & 2.30 & 2.55 \\
\hline & Allard $^{b}$ & 2600 & 3.5 & 0.0 & 12 & 0.229 & 1.90 & 1.56 & 1.73 \\
\hline & Allard $^{b}$ & 2600 & 4.0 & 0.0 & 12 & 0.229 & 1.76 & 1.44 & 1.25 \\
\hline & Allard $^{b}$ & 2800 & 4.0 & 0.0 & 12 & 0.265 & 2.84 & 2.20 & 1.91 \\
\hline & measured & & & & & & $3.2 \pm 2.3$ & $1.9^{1}$ & $2.9^{1}$ \\
\hline
\end{tabular}

a) AMES-cond models from Allard et al. (2001).

b) AMES-dusty models from Allard et al. (2001).

c) L \& T cloud-free model from Burrows et al. (2006).

d) L \& T model with clouds from Burrows et al. (2006).

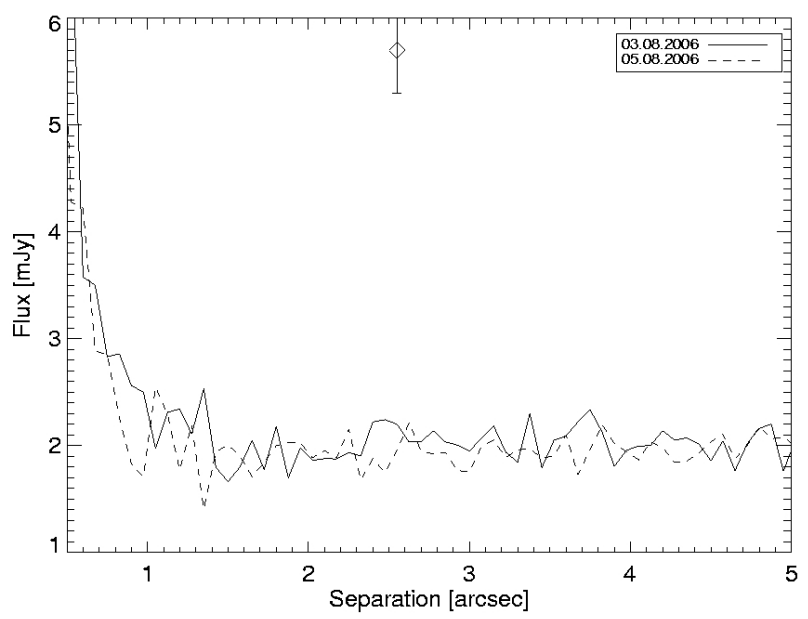

Fig. 3. Comparison of the limiting background obtained from the two data sets of HD 130948 taken in the SIV filter. The over-plotted point corresponds to the detection on the 5th of August, with a flux of $5.7 \mathrm{mJy}$.

GJ 229 B: Saumon et al. (2000), have used high-resolution infrared spectra to determine the metallicity, effective temperature and gravity of the T dwarf (see also Table 1). While using an age of 0.2 Gyr they derived an effective temperature of $950 \mathrm{~K} \pm 80 \mathrm{~K}$ and a gravity of $\log g=5 \pm 0.5$. Later on, Leggett et al. (2002) compared the observed low- and high- resolution spectra of GJ 229 A and GJ 229 B to theoretical spectra (AMES-models). Their best fit yields an $T_{\text {eff }}=1000 \pm 100$ and a gravity of $\log g \leq 3.5$ for GJ $229 \mathrm{~B}$, as well as a metallicity
1) Upper limit, limiting background plus $3 \sigma$.

2) Flux of one component assuming both L4 dwarfs contribute equally to the measured flux.

3) Only 05.08.2006.

of $[M / H] \approx-0.5$ and an age of $\sim 30 \mathrm{Myr}$ (range 16-45 Myr) for the system. While the metallicity is determined within the spectra fitting procedure, the age is derived by a comparison with evolutionary models, and mainly constrained by the observed luminosity and the derived effective temperature of the A component. Using VISIR mid-IR photometry, absolute model predictions of both Allard et al. (2001) and Burrows et al. (2006) can then be tested for different combinations of $T_{\text {eff }},[M / H]$ and gravity (when available, e.g. see Fig. 4). From Table 5, we see that the PAH1, SIV and PAH2 photometry are consistent with model predictions for a $T_{\text {eff }}=900 \mathrm{~K}, \log g=5.0$ and $[M / H]=$ 0 companion. At solar metallicity, an effective temperature of $T_{\text {eff }}=1000 \mathrm{~K}$ can be excluded at more than 2 sigma. At subsolar $[M / H]=-0.5$ metallicity, low gravity $(\log g<4.5)$ values remain excluded for $T_{\text {eff }}=1000 \mathrm{~K}$. Therefore, excluding young ages predictions of Leggett et al. (2002), the VISIR photometry clearly favours the initial physical parameters proposed by Saumon et al. (2000) for solar and subsolar metallicities.

HD 130948 BC: As already mentioned, HD $130948 \mathrm{BC}$ is a binary brown dwarf consisting of two L 4 dwarfs. To compare our observations to the model predictions, we assumed that both brown dwarfs contribute equally to the measured fluxes, as the simplest assumption. An unequal distribution of the measured flux on one of the two brown dwarfs would only increase the afterwards described effect. The suggested effective temperature of $\sim 1900 \mathrm{~K}$ places the binary in the regime of the AMESdusty models. While the predicted PAH1 flux is in good agreement with our measurements, most models fail to reproduce the SIV flux detected on August 5, 2006, but would be consistent with the non-detection on August 3, 2007. Using the 


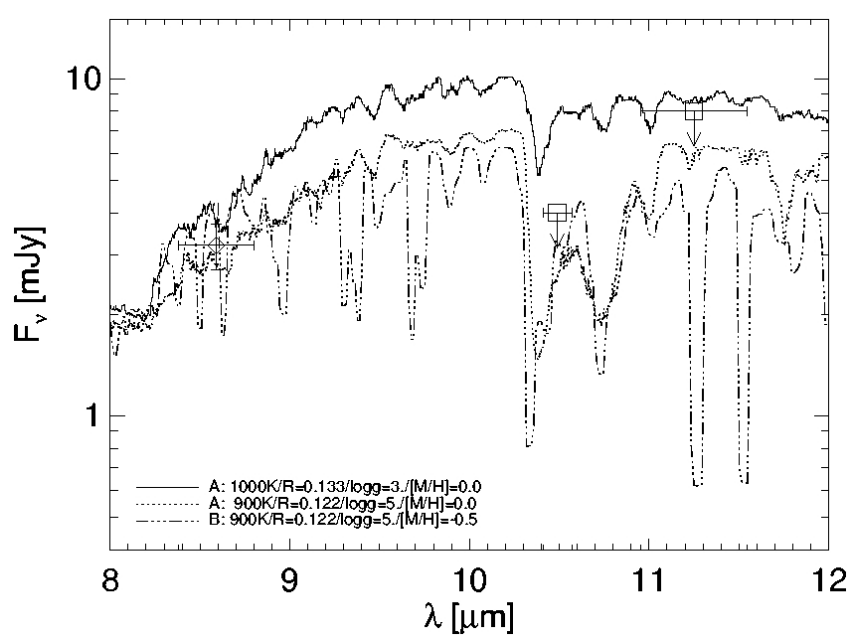

Fig. 4. GJ 229 B: comparison of theoretical spectra from Allard et al. (2001) and Burrows et al. (2006) with the VISIR photometry.

models provided by Burrows et al. (2006) we tested the influence of different metallicities $([M / H]=0.0$ and $[M / H]=-0.5)$ on the predicted fluxes. The change is marginal, only about $0.05 \mathrm{mJy}$.

\subsection{HD 130948BC: photometric variability}

While analysing the SIV data obtained for HD 130948, we found that the binary companion was detectable in only one of the two data sets. A possible explanation of this result is an intrinsic variability around $10.5 \mu \mathrm{m}$ of one or both of the $\mathrm{L}$ dwarfs in the binary. Variability at $10.5 \mu \mathrm{m}$ could either be caused by ammonia $\left(\mathrm{NH}_{3}\right)$ or silicates. Since the $\mathrm{NH}_{3}$ absorption features, which were first identified in the mid-infrared spectra taken with Spitzer/IRAS by Roellig et al. (2004), and Cushing et al. (2006), appear at roughly the $\mathrm{L} / \mathrm{T}$ transition this is unlikely to be the cause of the observed variability. A more favourable explanation may be an inhomogeneous distribution of silicate clouds, which characterise the atmospheres of $\mathrm{L}$ dwarfs with effective temperatures of roughly 1400-2000 K (Burrows et al. 2001). Future VISIR observations of HD $130948 \mathrm{BC}$ at $10.5 \mu \mathrm{m}$ over different timescales should secure this photometric variability.

\section{Summary}

Using VISIR at the VLT, we performed a mini-survey of brown dwarfs in binary systems. The four selected brown dwarfs were imaged in three narrow band filters at 8.6, 10.5 and $11.25 \mu \mathrm{m}$. At $8.6 \mu \mathrm{m}$ three of the brown dwarfs were detected and photometry was obtained. None of the brown dwarfs was detected at $11.25 \mu \mathrm{m}$, and only HD $130948 \mathrm{BC}$ was detected at $10.5 \mu \mathrm{m}$. The observations of HD $130948 \mathrm{BC}$ at $10.5 \mu \mathrm{m}$ indicate a possible variation of one or both brown dwarfs of the binary.
To constrain the atmospheric properties of the brown dwarfs we compared the mid-infrared photometry to theoretical model spectra by Allard et al. (2001) and Burrows et al. (2006). The measured mid-infrared fluxes and upper limit of GJ 229 B are consistent with the characteristic parameter obtained, by Saumon et al. (2000) $\left(T_{\text {eff }} \sim 950 \mathrm{~K}, \log g \sim 5\right.$, respectively), while values of the effective temperature and gravity as suggested by Leggett et al. (2002) $\left(T_{\text {eff }} \sim 1000 \mathrm{~K}\right.$, $\log g \leq 3.5$, respectively) result in too high model fluxes. As for HD $130948 \mathrm{BC}$, the model fluxes for $T_{\mathrm{eff}} \sim 1900 \mathrm{~K}, \log g \leq 5$ fit the measurement at $8.6 \mu \mathrm{m}$ and the upper limits obtained at $10.5 \mu \mathrm{m}$ and $11.25 \mu \mathrm{m}$. Nevertheless, the models are not in agreement with the flux measured for the detection of HD $130948 \mathrm{BC}$ at $10.5 \mu \mathrm{m}$ during one observing epoch.

\section{References}

Allard, F., Hauschildt, P. H., Baraffe, I., \& Chabrier, G. 1996, ApJ, 465, L123 Allard, F., Hauschildt, P. H., Alexander, D. R., et al. 2001, ApJ, 556, 357 Burgasser, A. J., Kirkpatrick, J. D., Cutri, R. M., et al. 2000, ApJ, 531, L57 Burrows, A., Marley, M., Hubbard, W. B., et al. 1997, ApJ, 491, 856 Burrows, A., Hubbard, W. B., Lunine, J. I., et al. 2001, RvMP, 73, 719 Burrows, A., Sudarsky, D., \& Hubeny, I. 2006, ApJ, 640, 1063 Chen, B., Asiain, R., Figueras, F., et al. 1997, A\&A, 318, 29 Cohen, M., Walker, R. G., Carter, B., et al. 1999, AJ, 117, 1864 Cushing, M. C., Roellig, Th. L., Marley, M. S., et al. 2006, ApJ, 648, 614 Cutri, R. M., Skrutskie, M. F., van Dyk, S., et al. 2003, 2MASS All-Sky Catalog of Point Sources

Epchtein, N., de Batz, B., Capoani, L., et al. 1997, The Messenger, 87, 27 Fuhrmann, K. 2004, AN, 325, 1, 3

Gaidos, E. J. 1998, PASP, 110, 1259

Gaidos, E. J., Henry, G. W., \& Henry, S. M. 2000, AJ, 120, 1006 Golimowski, D. A., Burrows, C. J., Kulkarni, S. R., et al. 1998, AJ, 115, 2579 Goto, M., Kobayashi, N., \& Terada, H. 2002, ApJ, 567, L59

Guenther, E. W., Neuhäuser, R., Huélamo, N., et al. 2001, A\&A, 365, 514 Lagage, P. O., Pel, J. W., Authier, M., et al. 2004, The Messenger, 117, 12 Leggett, S. K., Toomey, D. W., Geballe, T. R., et al. 1999, ApJ, 517, L139 Leggett, S. K., Hauschildt, P. H., Allard, F., et al. 2002 MNRAS, 332, 78 Leggett, S. K., Saumon, D., Marley, M. S., et al. 2007, ApJ, 655, 1079

Liu, M. C., Fischer, D. A., Graham, J. R., et al. 2002, ApJ, 571, 519 Lowrance, P. J., Schneider, G., Kirkpatrick, J. D., et al. 2000, ApJ, 541, 390 Mainzer, A. K., Roellig, Th. L., Saumon, D., et al. 2007 [arXiv: astro-ph/0701398]

Marley, M. S., Saumon, D., Guillot, T., et al. 1996, Science, 272, 1919 Matthews, K., Nakajima, T., Kulkarni, S. R., et al. 1996, AJ, 112, 1678 Nakajima, T., Oppenheimer, B. R., Kulkarni, S. R., et al. 1995, Nature, 378, 463 Pantin, E., Vanzi, L., \& Weilenman, U. 2007, to appear in the proceedings of the ESO Instrument Calibration Workshop

Potter, D., Martin, E. L., Cushing, M. C., et al. 2002, ApJ, 567, L133 Roellig, T. L., van Cleve, J. E., Sloan, G. C., et al. 2004, ApJS, 154, 418 Saumon, D., Geballe, T. R., Leggett, S. K., et al. 2000, ApJ, 541, 374 Saumon, D., Marley, M. S., Cushing, M. C., et al. 2006, ApJ, 647, 552 Soderblom, D. R., \& Mayor, M. 1993, AJ, 105, 226

Sterzik, M. F., Pantin, E., Hartung, M., et al. 2005, A\&A, 436, L39 Stoughton, C., Lupton, R. H., Bernardi, M., et al. 2002, AJ, 123, 485

Valenti, J. A., \& Fischer, D. A. 2005, ApJS, 159, 141

Zuckerman, B., Song, I., Bessell, M. S. et al. 2001, ApJ, 562, L87 